\title{
Regioselective Synthesis of Spiro-2-aminopyrimidinone Derivatives in Ionic Liquid as Green Solvent
}

\author{
U. S. SISODIA, S. KHOTHARI and S. AMETA \\ Pacific University, Udaipur, Rajasthan-313024, India \\ seemavkothari@rediffmail.com
}

Received 2 July 2016 / Accepted 10 October 2016

\begin{abstract}
An interesting regioselectivity was investigated for the synthesis of spiro-2aminopyrimidinones-5-carbonitrile (4a-e) by the multicomponent condensation of cyclic ketones (1a-d), alkyl cyanoacetates (2) and various guanidine salts (3) using 3-butyl-1-methyl-1H-imidazol-3ium tetrafluoroborate $\left([\mathrm{BMIM}]\left[\mathrm{BF}_{4}\right]\right)$ as ionic liquid. Existence of two possible potentially active centers i.e. $-\mathrm{CN}$ and $-\mathrm{COOR}$ groups in the intermediate to Michael adduct results in possibility of formation of two region-isomeric products i.e. spiro-2-aminopyrimidinones-5-carbonitrile (4a-e) or spiro-2-amino-4-imino-pyrimidin-5-carboxylic acid alkyl ester (5). High regioselectivity was displayed with exclusive formation of compound (4a-e) which was further confirmed by spectral studies.
\end{abstract}

Keywords: Regioselectivity, Spiro-2-aminopyrimidinones-5-carbonitrile, Ionic liquid, Green solvent

\section{Introduction}

Recently, the growing awareness of environmental issues has focused attention on the need for greener and more sustainable technologies in the chemical industry ${ }^{1}$. The number of environmental laws and regulations has increased over the years and more specific regulations have been set in order to provide a safer environment. Thus the chemistry community has been mobilized to develop new chemistries that are less hazardous to human health and the environment. Green chemistry is not a part of the large field of chemistry, but it is a way of thinking and doing things better and more efficiently, because solvents are often necessary in chemical reactions, alternative solvents have been developed. The ideal solvent should have very low volatility, and it should be chemically and physically stable, recyclable, reusable and easy to handle. Therefore, one such candidate is an ionic liquid $(\mathrm{IL})^{2}$, which consist of cations and anions, have become an interesting research topic in the last decade, which is available in some books ${ }^{3,4}$ and reviews ${ }^{5,6}$ published about them. Many different ionic liquids have been prepared and they have successfully used as solvents in diverse reactions, such as Diels-Alder ${ }^{7}$, Friedel-Crafts ${ }^{8}$, Heck $^{9}$, hydration ${ }^{10}$, oxidation ${ }^{11}$, alkylation $^{12}$, allylation ${ }^{13}$, hydroformulation ${ }^{14}$, esterification ${ }^{15}$, dimerization ${ }^{16}$ and polymerization reactions ${ }^{17}$ and enzyme catalysis ${ }^{18}$, often leading to better selectivity, yield and reaction rates than with volatile organic solvents. ILs have a great variety of chemical and physical properties that can be tuned with cations and anions ${ }^{19}$. 
Organic molecules containing a spiro heterobicyclic moiety are of broad scientific interest due to their unique chemical and conformational features as well as the biological properties often associated with the asymmetric spiro carbon atom. They have attracted considerable attention from the synthetic community ${ }^{20}$. For example, new class of marine toxins isolated from shellfish and dinoflagellate, such as pinnatoxins and pteriatoxin ${ }^{21}$, exhibits an azaspiro system responsible for the biological activity.

In addition, many spiro-compounds possess very promising biological activities as anticancer agents $^{22}$, antibacterial agents ${ }^{23}$, anticonvulsant agents ${ }^{24}$, anti-tuberculosis agents ${ }^{25}$, anti-Alzheimer's agents ${ }^{26}$, pain-relief agents ${ }^{27}$, anti-dermatitis agents ${ }^{28}$ and antimicrobial agents ${ }^{29}$. In addition to their medical uses, some spiro-compounds have found other uses in the agricultural and industrial fields. For example, they are used as antifungal agents ${ }^{30}$, pesticides $^{31}$, laser dyes ${ }^{32}$ and electroluminescent devices ${ }^{33}$. Spiro- compounds have also been recently used as antioxidants ${ }^{34}$. Hence, the synthesis of spiro-compounds incorporating privileged heterocycles could be a valuable strategy to discover new bioactive compounds in the context of chemical genomics.

\section{Experimental}

All the synthesized compounds were subjected to various physicochemical measurements. Molecular weights were determined by rast camphor method. Carbon and hydrogen analysis of the compounds done at the microanalytical laboratory, Department of Chemistry, Punjab University, Chandigarh. IR spectra were recorded on a Nicolet Megna FTIR-550 spectrophotometer using $\mathrm{KBr}$ pellets. The ${ }^{1} \mathrm{H}$ NMR and ${ }^{13} \mathrm{C}$ NMR spectra were recorded on Bruker DRX-300 NMR spectrometer at $300 \mathrm{MHz}$ and $75 \mathrm{MHz}$ respectively in DMSO- $\mathrm{d}_{6}$ using trimethyl silane as internal standard. Mass spectrums of representative compounds were recorded on Kratos 50 mass spectrometer at $70 \mathrm{eV}$. All the melting points were determined using open-ended capillary tube method.

\section{Regioselective synthesis of spiro-2-aminopyrimidine derivatives (4a-e)}

The synthesis of spiro-2-aminopyrimidine derivatives, In the ionic liquid procedure for the preparation of spiro derivatives, first step involves in situ formation of alkene derivative (6) by the knoevenagel condensation of cyclic ketone (1a-e) $(1 \mathrm{mmol})$ and alkyl cyanoacetates (2) (1 mmol). In the next step the formation of spiro derivatives (4a-e) was proceed via michael attack of free guanidine on $\mathbf{6}$ having electron attracting group on exomethylene carbon and afforded Micheal adduct (7). The intermediate Michael adducts which has two possible nucleophilic groups (i.e. -CN and -COOR). This would lead to the formation of either spiro-2-aminopyrimidinones-5-carbonitrile (4a-e) or spiro-2-amino-4imino-pyrimidin-5-carboxylic acid alkyl ester (5) subsequently. The reaction has been carried out in $25 \mathrm{~mL}$ ionic liquid [BMIM] [BF $]$. Reaction was completed in 2-3 $\mathrm{h}$ at $30-35{ }^{\circ} \mathrm{C}$. The progress of the reaction was monitored by TLC (hexane: ethylacetate 10:1). After completion of reaction, the reaction mixture was cooled to $30{ }^{\circ} \mathrm{C}$ and then the reaction mixture was poured into ice-cold water, neutralized by $1: 1 \mathrm{HCl}$ solution to get the desired product. The separated solid was filtered by whatman filter paper under vacuum and washed with little amount of distilled water to remove acid. Desired product obtained as a solid with $82-87 \%$ yield, solid was crystallized by ethanol. Filtrate was completely distilled out at $80-85{ }^{\circ} \mathrm{C}$ under reduce pressure of $600 \mathrm{~mm} \mathrm{Hg}$ to recycle ionic liquids which will be used for the synthesis of another compounds. The structures and reaction scheme of compounds (4a-e) are shown in Scheme 1 and 2. 
<smiles>CCCC(=O)CC[CH+]C(=O)OCC</smiles><smiles>CCCCC(C)N</smiles>
3<smiles>O=C1CCCCC1</smiles><smiles>O=C1CCCC1</smiles><smiles>O=C1CCCCC1</smiles>

b

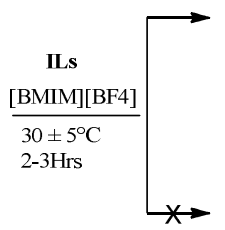<smiles>N#CC1C(=O)N=C(N)NC12CC2</smiles><smiles>[R]OC(=O)C1C(=N)N=C(N)NC12CC2</smiles>

Scheme 1. Regioselective synthesis of spiro-2-aminopyrimidine derivatives (4a-e)

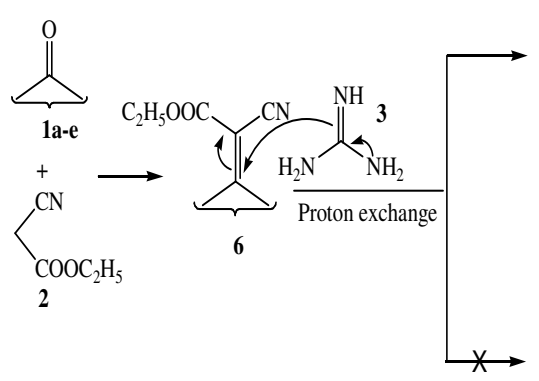<smiles>CCOC(=O)C1CCCC1(CC)NC(=N)N</smiles>

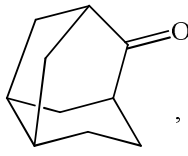

d<smiles>CC(C)(C)OC(=O)N1CCC(=O)CC1</smiles>

e

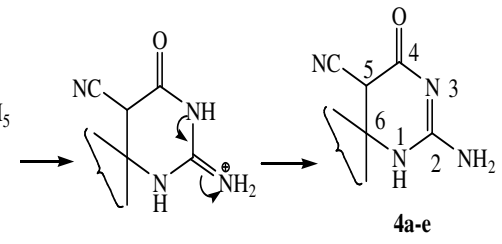<smiles>N#CCC1CCCC1(CC#N)NC(=N)N</smiles>

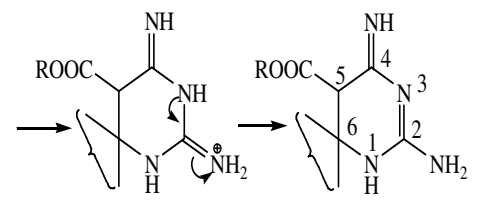

5

Scheme 2. Possible mechanisms of spiro-2-aminopyrimidine derivatives (4a-e)

\section{Results and Discussion}

The physical properties and analytical data of all the spiro-2-aminopyrimidine derivatives, are enlisted in Table 1. We have observed that the yield of compounds which contain electron withdrawing group is more than compounds having electron donating group. The time utilized for the synthesis of compounds using ionic liquid as a media is less as compared to commercial hazards solvents. Also the isolation of prepared compounds is more easy and eco-friendly in ionic liquid as compare to other hazards solvents. All the synthesized compounds are stable at ambient temperature, slightly soluble in ethanol, methanol and very soluble in DMF and DMSO. 
Table 1. Analytical data and physical properties of the compounds synthesized by IL method

\begin{tabular}{|c|c|c|c|c|c|c|}
\hline \multirow{2}{*}{ Compounds } & \multirow{2}{*}{$\begin{array}{c}\text { Melting } \\
\text { point, } \\
{ }^{0} \mathrm{C}\end{array}$} & \multirow{2}{*}{$\begin{array}{l}\text { Cyclic } \\
\text { ketones }\end{array}$} & \multicolumn{3}{|c|}{ Found (Calculated) $(\%)$} & \multirow{2}{*}{$\begin{array}{c}\text { Melting } \\
\text { point, } \\
{ }^{0} \mathrm{C}\end{array}$} \\
\hline & & & $\mathrm{C}$ & $\mathrm{H}$ & $\mathrm{N}$ & \\
\hline $\begin{array}{l}{[\mathrm{BMIM}]\left[\mathrm{BF}_{4}\right]} \\
\left(\mathrm{C}_{8} \mathrm{H}_{15} \mathrm{~F}_{4} \mathrm{~N}_{2} \mathrm{~B}\right)\end{array}$ & - & & $\begin{array}{c}68.90 \\
(69.02)\end{array}$ & $\begin{array}{c}10.72 \\
(10.86)\end{array}$ & $20.44(20.12)$ & $(139.22)$ \\
\hline $\begin{array}{c}\mathbf{4 a} \\
\left(\mathrm{C}_{9} \mathrm{H}_{12} \mathrm{~N}_{4} \mathrm{O}\right)\end{array}$ & $\begin{array}{l}282- \\
284\end{array}$ & & $\begin{array}{c}56.40 \\
(56.24)\end{array}$ & $\begin{array}{c}6.31 \\
(6.20)\end{array}$ & $29.24(28.26)$ & (192.22) \\
\hline $\begin{array}{c}\mathbf{4 b} \\
\left(\mathrm{C}_{10} \mathrm{H}_{14} \mathrm{~N}_{4} \mathrm{O}\right)\end{array}$ & $\begin{array}{c}298- \\
300\end{array}$ & & $\begin{array}{c}58.41 \\
(60.20)\end{array}$ & $\begin{array}{c}6.81 \\
(6.74)\end{array}$ & $27.25(27.79)$ & $(206.24)$ \\
\hline $\begin{array}{c}\mathbf{4 c} \\
\left(\mathrm{C}_{11} \mathrm{H}_{16} \mathrm{~N}_{4} \mathrm{O}\right)\end{array}$ & $\begin{array}{l}310- \\
312\end{array}$ & & $\begin{array}{c}59.79 \\
(59.98)\end{array}$ & $\begin{array}{c}7.34 \\
(7.32)\end{array}$ & $25.52(25.44)$ & $(220.27)$ \\
\hline $\begin{array}{c}\mathbf{4 d} \\
\left(\mathrm{C}_{14} \mathrm{H}_{18} \mathrm{~N}_{4} \mathrm{O}\right)\end{array}$ & $\begin{array}{l}280- \\
282\end{array}$ & & $\begin{array}{c}65.28 \\
(65.09)\end{array}$ & $\begin{array}{c}7.04 \\
(7.02)\end{array}$ & $21.75(21.69)$ & $(258.32)$ \\
\hline $\begin{array}{c}\mathbf{4 e} \\
\left(\mathrm{C}_{14} \mathrm{H}_{21} \mathrm{~N}_{5} \mathrm{O}_{3}\right)\end{array}$ & $\begin{array}{l}260- \\
262\end{array}$ & & $\begin{array}{c}54.54 \\
(54.71)\end{array}$ & $\begin{array}{c}6.86 \\
(6.89)\end{array}$ & $22.72(22.79)$ & $(307.35)$ \\
\hline
\end{tabular}

\section{Spectral data}

7-Amino-9-oxo-6,8-diaza-spiro [4.5] dec-7-ene-10-carbonitrile (4a)

$\mathrm{IR}\left(\mathrm{KBr}\right.$ pellet, $\left.\mathrm{cm}^{-1}\right): 3325-3134(\mathrm{NH} \& \mathrm{NH} 2), 2174(\mathrm{C} \equiv \mathrm{N}), 1676(\mathrm{C}=\mathrm{O}), 1585(\mathrm{C}=\mathrm{N}) .{ }^{1} \mathrm{H}$ NMR (400 MHz, $\left.\mathrm{CDCl}_{3}\right): \delta=1.28-1.40(\mathrm{~m}, 2 \mathrm{H}, \mathrm{CH} 2), 1.52-1.71(\mathrm{~m}, 4 \mathrm{H}, \mathrm{CH} 2), 1.80-1.96$ $(\mathrm{m}, 2 \mathrm{H}, \mathrm{CH} 2), 3.80(\mathrm{~s}, 1 \mathrm{H}, \mathrm{CH}), 6.86(\mathrm{~s}, 2 \mathrm{H}, \mathrm{NH} 2, \mathrm{D} 2 \mathrm{O}$ exchangeable), $7.58(\mathrm{~s}, 1 \mathrm{H}, \mathrm{NH}$, D2O exchangeable). ${ }^{13} \mathrm{C}$ NMR (100 MHz, $\left.\mathrm{CDCl}_{3}\right): 24.62$ (C'1), 28.83 (C'4), 32.26 (C'3), $62.84(\mathrm{C} 5-\mathrm{CN}), 52.83$ (spiro carbon), $115.48(\underline{\mathrm{CN}}), 166.17\left(\underline{\mathrm{C}}_{2}-\mathrm{NH}_{2}\right), 182.62(\underline{\mathrm{C}}=\mathrm{O})$,

MS: $m / z: 193.56$

\section{2-Amino-4-oxo-1,3-diaza-spiro [5.5] undec-2-ene-5-carbonitrile (4b)}

$\operatorname{IR}\left(\mathrm{KBr}\right.$ pellet, $\left.\mathrm{cm}^{-1}\right): 3320-3130(\mathrm{NH} \& \mathrm{NH} 2), 2170(\mathrm{C} \equiv \mathrm{N}), 1670(\mathrm{C}=\mathrm{O}), 1590(\mathrm{C}=\mathrm{N}) .{ }^{1} \mathrm{H}$ NMR (400 MHz, $\mathrm{CDCl}_{3}$ ): 1.18-1.23 (m, 2H, CH2), 1.38-1.51 (m, 2H, CH2), 1.58-1.61 (m, $6 \mathrm{H}, \mathrm{CH} 2), 3.88$ (s, 1H, CH), 6.92 (s, 2H, NH2, D2O exchangeable), 7.65 (s, 1H, NH, D2O exchangeable). ${ }^{13} \mathrm{C}$ NMR (100 MHz, $\left.\mathrm{CDCl}_{3}\right): 20.4\left(\underline{\mathrm{C}}^{\prime}{ }_{1}\right), 21.64\left(\underline{\mathrm{C}}_{5}^{\prime}\right), 24.8\left(\underline{\mathrm{C}}_{4}{ }_{4}\right), 28.74\left(\underline{\mathrm{C}}_{3}{ }_{3}\right)$, $34.29\left(\underline{\mathrm{C}}_{2}^{\prime}\right), 54.22$ (spiro carbon), $61.87\left(\underline{\mathrm{C}}_{5}-\mathrm{CN}\right), 118.45(\underline{\mathrm{CN}}), 170.11\left(\underline{\mathrm{C}}_{2}-\mathrm{NH}_{2}\right), 183.85$ $(\underline{\mathrm{C}}=\mathrm{O})$. MS: $m / z: 207.77$ 


\section{2-Amino-9-methyl-4-oxo-1,3-diaza-spiro [5.5] undec-2-ene-5-carbonitrile (4c)}

IR $\left(\mathrm{KBr}\right.$ pellet, $\left.\mathrm{cm}^{-1}\right): 3324-3132\left(\mathrm{NH} \& \mathrm{NH}_{2}\right), 2174(\mathrm{C} \equiv \mathrm{N}), 1668(\mathrm{C}=\mathrm{O}), 1592(\mathrm{C}=\mathrm{N}) .{ }^{1} \mathrm{H}$ NMR (400 MHz, $\left.\mathrm{CDCl}_{3}\right): 1.22-1.28\left(\mathrm{q}, 3 \mathrm{H}, \mathrm{CH}_{3}\right), 1.54-1.68\left(\mathrm{~m}, 4 \mathrm{H}, \mathrm{CH}_{2}\right), 1.74-1.84(\mathrm{~m}$, $\left.4 \mathrm{H}, \mathrm{CH}_{2}\right), 1.88-1.98(\mathrm{~m}, 1 \mathrm{H}, \mathrm{CH}), 3.90(\mathrm{~s}, 1 \mathrm{H}, \mathrm{CH}), 6.96\left(\mathrm{~s}, 2 \mathrm{H}, \mathrm{NH}_{2}, \mathrm{D}_{2} \mathrm{O}\right.$ exchangeable), 7.68 (s, $1 \mathrm{H}, \mathrm{NH}, \mathrm{D}_{2} \mathrm{O}$ exchangeable). ${ }^{13} \mathrm{C}$ NMR $\left(100 \mathrm{MHz}, \mathrm{CDCl}_{3}\right): 16.82\left(\mathrm{CH}_{3}\right), 26.46\left(\underline{\mathrm{C}}^{\prime}\right)$, $29.68\left(\underline{\mathrm{C}}_{5}^{\prime}\right), 34.87\left(\underline{\mathrm{C}}_{4}^{\prime}\right), 34.94\left(\underline{\mathrm{C}}^{\prime}{ }_{2}\right), 38.25\left(\underline{\mathrm{C}}_{3}{ }_{3}\right), 58.22$ (spiro carbon), $64.54\left(\underline{\mathrm{C}}_{5}-\mathrm{CN}\right)$, $122.4(\underline{\mathrm{CN}}), 176.12\left(\underline{\mathrm{C}}_{2}-\mathrm{NH}_{2}\right), 190.85(\underline{\mathrm{C}}=\mathrm{O})$.

MS: $m / z: 221.17$.

2-Amino-6-oxo-2,3,3a,4,5,5',6,6',7,7a-decahydro-1H,3H-spiro[2,5-methanoindene8,4-pyrimidine]-5-carbonitrile $(\mathbf{4 d})$

$\operatorname{IR}\left(\mathrm{KBr}\right.$ pellet, $\left.\mathrm{cm}^{-1}\right): 3330-3138\left(\mathrm{NH} \& \mathrm{NH}_{2}\right), 2186(\mathrm{C} \equiv \mathrm{N}), 1672(\mathrm{C}=\mathrm{O}), 1592(\mathrm{C}=\mathrm{N}) .{ }^{1} \mathrm{H}$ NMR (400 MHz, $\left.\mathrm{CDCl}_{3}\right): 1.58-1.69\left(\mathrm{~m}, 8 \mathrm{H}, \mathrm{CH}_{2}\right), 1.72-1.88(\mathrm{~m}, 5 \mathrm{H}, \mathrm{CH}), 3.96(\mathrm{~s}, 1 \mathrm{H}, \mathrm{CH})$, 6.98 (s, 2H, NH $\mathrm{N}_{2} \mathrm{D}_{2} \mathrm{O}$ exchangeable), 7.86 (s, $1 \mathrm{H}, \mathrm{NH}, \mathrm{D}_{2} \mathrm{O}$ exchangeable). ${ }^{13} \mathrm{C}$ NMR (100 $\left.\mathrm{MHz}, \mathrm{CDCl}_{3}\right): 24.4,26.4,27.4,32.6,38.8,44.2$, (aliphatic- $\underline{\mathrm{C}}$ ), $63.62\left(\underline{\mathrm{C}}_{5}-\mathrm{CN}\right), 60.28$ (spiro carbon), $128.49(\underline{\mathrm{CN}}), 180.94\left(\underline{\mathrm{C}}_{2}-\mathrm{NH}_{2}\right), 192.64(\underline{\mathrm{C}}=\mathrm{O})$.

MS: $m / z: 259.46$.

2-Amino-5-cyano-4-oxo-1,3,9-triaza-spiro [5.5]undec-2-ene-9-carboxylic acid tertbutyl ester (4e)

$\mathrm{IR}\left(\mathrm{KBr}\right.$ pellet, $\left.\mathrm{cm}^{-1}\right): 3330-3140\left(\mathrm{NH} \& \mathrm{NH}_{2}\right), 2180(\mathrm{C} \equiv \mathrm{N}), 1680-1700$ two $(\mathrm{C}=\mathrm{O}), 1598$ $(\mathrm{C}=\mathrm{N}) .{ }^{1} \mathrm{H}$ NMR $\left(400 \mathrm{MHz}, \mathrm{CDCl}_{3}\right): 1.47\left(\mathrm{~s}, 9 \mathrm{H}, \mathrm{C}\left(\mathrm{CH}_{3}\right)_{3}\right), 1.68-1.88\left(\mathrm{~m}, 4 \mathrm{H}, \mathrm{CH}_{2}\right), 2.08-$ $2.32\left(\mathrm{~m}, 2 \mathrm{H}, \mathrm{CH}_{2}\right), 3.98(\mathrm{~s}, 1 \mathrm{H}, \mathrm{CH}), 6.96\left(\mathrm{~s}, 2 \mathrm{H}, \mathrm{NH}_{2}, \mathrm{D}_{2} \mathrm{O}\right.$ exchangeable), $7.78(\mathrm{~s}, 1 \mathrm{H}, \mathrm{NH}$, $\mathrm{D}_{2} \mathrm{O}$ exchangeable. ${ }^{13} \mathrm{C}$ NMR $\left(100 \mathrm{MHz}, \mathrm{CDCl}_{3}\right): 20.27\left(\mathrm{CH}_{3}\right), 24.95\left(\underline{\mathrm{C}}\left(\mathrm{CH}_{3}\right)_{3}\right), 28.75$ $\left(\underline{\mathrm{C}}_{3}^{\prime}\right), 29.08\left(\underline{\mathrm{C}}_{5}^{\prime}\right), 37.08\left(\underline{\mathrm{C}}_{2}\right), 42.32\left(\underline{\mathrm{C}}_{6}^{\prime}\right), 54.43$ (spiro carbon), $68.21\left(\underline{\mathrm{C}}_{5}-\mathrm{CN}\right), 118.64$ $(\underline{\mathrm{CN}}), 166.65\left(\underline{\mathrm{C}}_{2}-\mathrm{NH}_{2}\right), 172.61(\underline{\mathrm{C}}=\mathrm{O}), 180.24\left(\underline{\mathrm{C}}_{4}=\mathrm{O}\right)$.

MS: $m / z: 308.67$

\section{Conclusion}

An interesting regioselectivity is investigated in the multicomponent condensation of cyclic ketones, alkyl cyanoacetates and various guanidine salts using ionic liquids (3-butyl-1methyl-1H-imidazol-3-ium tetrafluoroborate). Ionic liquid procedure resulted in the expected increment of yields, under ionic liquid the general applicability of this procedure is extended to a variety of ketones with high purity. Also addition of an ionic liquid increases the speed of reaction and reaction yields. It was possible to add catalytic quantities of ionic liquids in conventional solvent and still achieve a much greener reaction outcome. The prominent advantages of this methodology are reusability of the ionic liquids, operational simplicity, easy workup procedure, avoiding hazardous organic solvents and good yields of product.

\section{References}

1. Mestres R, Green Chem., 2004, 6, G10-12.

2. Seddon K R, Rogers R D and Volkov S, Green Industrial Applications of Ionic Liquids Proceedings of the NATO advanced research in Heraklion, Crete, Greece, Kluwer Academic Publishers, Dordrecht, Netherland, 2002.

3. Wassercheid P and Welton T, Ionic Liquids in Synthesis, Wiley-WCH, Weinheim, Germany, 2003.

4. Seddon K R and Rogers R D, Ionic Liquids as Green Solvents: Progress and Prospects (Proceedings of the $224^{\text {th }}$ ACS symposium in Boston 18-22 August 2002), ACS Symp Ser Washington D.C. 2003, 856. 
5. Scammels P J, Scott J L and Singer R D, Aust J Chem., 2005, 58(3), 155-169; DOI:10.1071/CH04272

6. Welton T, Coord Chem Rev., 2004, 248(21-24), 2459-2477; DOI:10.1016/j.ccr.2004.04.015

7. Jaeger D A and Tucker C E, Tetrahedron Lett., 1989, 30(15), 1785-1788; DOI:10.1016/S0040-4039(00)76362-3

8. Stark A, MacLean B L and Singer R D, J Chem Soc Dalton Trans., 1999, 63-66; DOI:10.1039/A806708B

9. Herrmann W A and Böhm V P W, Chem Eur J., 2000, 6, 1017-1025; DOI:10.1002/(SICI)1521-3765(20000317)6:6<1017::AID-CHEM1017>3.0.CO;2-8

10. Suarez P A Z, Dullius J E L, Einloft S, de Souza R F and Dupont J, Polyhedron, 1996, 15, 1217-1219; DOI:10.1016/0277-5387(95)00365-7

11. Howarth J, Tetrahedron Lett., 2000, 41, 6627-6629; DOI:10.1016/S00404039(00)01037-6

12. Badri M, Brunet J and Perron R, Tetrahedron Lett., 1992, 33(31), 4435-4438; DOI:10.1016/S0040-4039(00)60103-X

13. Gordon C M and McCluskey A, Chem Comm., 1999, 1431-1432; DOI:10.1039/A903661J

14. Wasserscheid P, Waffenschmidt H, Machnitzki P, Kottsieper K W and Stelzer O, Chem Comm., 2001, 451-452; DOI:10.1039/B009919H

15. Zim D, de Souza R F, Dupont J and Monteiro A L, Tetrahedron Lett., 1998, 39(39), 7071- 7074; DOI:10.1016/S0040-4039(98)01551-2

16. Chauvin Y, Gilbert B and Guilbard I, J Chem Soc Chem Comm., 1990, 1715-1716; DOI:10.1039/C39900001715

17. Carmichael A J, Haddleton D M, Bon S A F and Seddon K R, Chem Comm., 2000, 1237-1238; DOI:10.1039/B003335I

18. Schöfer S H, Kafzik N, Wasserscheid P and Kragl U, Chem Comm, 2001, 425-426; DOI:10.1039/B009389K

19. Anthony J L, Brennecke J F, Holbrey J D, Maginn E J, Mantz R A, Rogers R D, Trulove P C, Visser A E and Welton T, Physicochemiocal properties of ionic liquids. In Wassercheid $\mathrm{P} \&$ Welton $\mathrm{T}$ (Eds) Ionic Liquids in Synthesis, Wiley-WCH, Weinheim, 2003, 41-126.

20. Jadidi K, Ghahremanadeh R and Bazgir A, Tetrahedron, 65(10), 2009, 2005-2009; DOI:10.1016/j.tet.2009.01.013 (b) Pawar M J, Burungale A B and Karale B K, Arkivoc, 2009, 13, 97.

21. Brimble M A, Crimmins D and Trzoss M, Arkivoc, 2005, 1, 39.

22. Young-Won C, Angela S, Bao-Ning S, Qiuwen M, Hee-Byung C, Soedarsono R, Leonardus K, Agus R, Norman F, Steven S and Douglas K, J Nat Prod., 2008, 71(3), 390-395; DOI:10.1021/np070584j

23. Hyeong Beom P, Nam Hyun J, Joon Hee H, Jung Hoon C, Jung Hoon C, Jung-Hyuck C, Kyung Ho Y and Chang-Hyun O, Arch Pharm., 2007, 340(10), 530-357; DOI:10.1002/ardp.200700060

24. Jolanta O, Krzysztof K and Ewa T, Pharmacol Rep., 2006, 58, 207.

25. Chande M S, Verma R S, Barve P A, Khanwelkar R R, Vaidya R B and Ajaikumar K B, Eur J Med Chem., 2005, 40(11), 1143-1148; DOI:10.1016/j.ejmech.2005.06.004

26. Pradhan R, Patra M, Behera A K, Mishra B K and Behera R K, Tetrahedron, 2006, 62(5), 779-828; DOI:10.1016/j.tet.2005.09.039 
27. Whiteside G T, Harrison J E, Pearson M S, Chen Z, Rotshteyn Y, Turchin P I, J. D. Pomonis J D, Mark L, Walker K and Brogle K C, J Pharmacol Exp Ther., 2004, 310(2), 793-799; DOI:10.1124/jpet.103.063560

28. Nakao K, Ikeda K, Kurokawa T, Togashi Y, Umeuchi H, Honda T, Okano K and Mochizuki H, Nihon Shinkei SeishinYakurigaku Zasshi, 2008, 28(2), 75-83.

29. Thadhaney B, Sain D, Pernawat G and Talesara G L, Indian J Chem., 2010, 49B.

30. Hejiao H, Huijuan G, Erwei L, Xingzhong L, Yuguang Z and Yongsheng C, J Nat Prod., 2006, 69(12), 1672-1675; DOI:10.1021/np0603830

31. Wei R, Liu Y and Liang Y, Chin J Org Chem., 2009, 29(3), 476-487.

32. Sumi K and Konishi G, Molecules, 2010, 15(11), 7582-7592; DOI:10.3390/molecules15117582

33. Pudzich R, Fuhrmann-Lieker T and Salbeck J, Adv Polym Sci., 2006, 199, 83-142; DOI:10.1007/12_074

34. Sarma B K, Manna D, Minoura M and Mugesh G, J Am Chem., Soc., 2010, 132(15), 5364-5374; DOI:10.1021/ja908080u 